\title{
Intersubjetividad:
}

\section{Esencia humana del cuidado profesional de Enfermería}

Lic. Guadalupe Leticia Aparicio Santiago*, Lic. Alicia Hernández Cantoral* Mtra. Rosa María Ostiguín Meléndez**

* Alumnas de la cuarta generación del Programa de Maestría en Enfermería ENEO-UNAM.

** Tutora del Programa de Maestría en Enfermería ENEO- UNAM

Este trabajo pretende resaltar el fenómeno de intersubjetividad en la relación Enfermera persona; rescatando la concepción humanista de la práctica profesional del cuidado.

Debido a las múltiples visiones teóricas que existen entorno a la intersubjetividad el análisis de este trabajo se centra en la propuesta de Alfred Schütz, sobre la teoría de comprensión intersubjetiva, que se sitúa en el ámbito de las relaciones sociales, y que plantea la interacción sujeto - sujeto, donde ambos aprenden, comparten y reconocen su humanidad, que en el terreno de la Enfermería permite tener una visión del ser humano como persona única, singular e indivisible.

El fenómeno intersubjetivo, permite el encuentro con el otro u otros, de una forma más integral y humana, por lo que es un elemento indispensable en la relación Enfermera - persona, tal cual lo sustentan las teorías disciplinarias de H. Peplau, J. Paterson y L. Zderad, J. Travelbee, J. Watson y R. Parse, donde cada una plantea su propia perspectiva de intersubjetividad. Para ello el ensayo presenta de manera general algunos referentes del fenómeno de intersubjetividad desde la perspectiva de Alfred Schütz, para con ello analizar las teorías de Enfermería de H. Peplau, J. Paterson y L. Zderad, J. Travelbee, J. Watson y R. Parse, y reconocer aspectos de convergencia.

Palabras claves: intersubjetividad, cuidado humano, relación Enfermera persona.

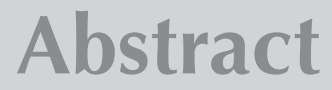

This work tries to emphasize the phenomenon of intersubjectivity in the relation person nurse; rescuing the conception humanist of the professional practice of the care. Due to the multiple theoretical visions that exist surroundings to the intersubjectivity the analysis of this work is centered in the proposal of Alfred Schütz, on the theory of intersubjective understanding, that it locates itself in the scope of the social relations, and which it raises the subject interaction - subject, where both learn, they share and they recognize his humanity, that in the land of the infirmary allow to have a vision of the human being like unique person, singular and indivisible.

The intersubjective phenomenon, allows the encounter with the other either others, of one more an integral and human form, reason why it is an indispensable element in the relation nurse - person, so as they sustain the disciplinary theories to it of H. Peplau, J. Paterson and L. Zderad, J. Travelbee, J. Watson and Even R., where each one raises its own perspective of intersubjectivity. For it the test presents/displays of general way some referring ones of the intersubjectivity phenomenon from the perspective of Alfred Schütz, towards it to analyze theories of infirmary of H. Peplau, J. Paterson and L. Zderad, J. Travelbee, J. Watson and Even $R$. and to recognize aspects of convergence.

Key words: Intersubjectivity, human care, relation person nurse. 


\section{CONCEPCIÓN HUMANISTA DEL CUIDADO}

Enfermería tiene como sujeto de su conocimiento y praxis al hombre; cuida la vida y la salud de la persona, además emplea las interrelaciones humanas en su práctica, por lo que debe considerarse y cultivarse como una ciencia humana, lo que implica un enfoque individual dirigido a la persona que integra todas las partes de un todo unificado y significativo. ${ }^{1}$

Desde este punto de vista la concepción humanista se centra en la persona, en su visión de ser único, integral, trascendental, lo que permite establecer su relación con las ciencias que se responsabilizan del cuidado del hombre, de la vida humana y de la salud, como lo es Enfermería. Esta visión abre el camino a una práctica que va más allá de un simple contacto físico y hace necesario utilizar elementos como la intersubjetividad, para rescatar la esencia humana del cuidado.

Quien más ha trabajado sobre el cuidado humanizado es Jean Watson, que en una de sus obras (1983) refuerza los lineamientos que conllevan a brindar cuidado humanista; estos postulados comprenden: tratar al individuo como persona, tener preocupación y empatía hacia él, donde influyen características personales de la Enfermera, un esfuerzo bilateral y un proceso de comunicación eficaz. ${ }^{2}$ Dentro de este proceso de comunicación interviene la relación intersubjetiva, donde convergen dos realidades que dan significado a su vida.

\section{Antecedentes del concepto de intersubjetividad}

Hablar de comunicación en el cuidar, implica utilizar elementos que favorezcan el conocer al otro y conocerse uno mismo a través de él, esto es aludir a la intersubjetividad entendida como elemento indispensable, para compartir un conocimiento con el otro. ${ }^{3}$

Para entender a la intersubjetividad se debe clarificar la noción de "subjetividad". La subjetividad se refiere a la conciencia que se tiene de todas las cosas desde el punto de vista propio, que se comparte colectivamente en la vida cotidiana; involucra un proceso interpersonal en el que los participantes expresan algo de si mismos, con la intención de influir de algún modo en la conducta del otro" ${ }^{4}$, que en el campo de la Enfermería tiene particular sentido en la relación Enfermera persona, ya que continuamente la Enfermera y la persona establecen un contacto directo, ya sea para brindar apoyo, ayuda o compañía.

Múltiples sociólogos han planteado la interacción como un eje para las relaciones sociales, tal es el caso de Habermas, Trevarthen y Schütz, sin embargo este último resulta ser el más pertinente para hablar de intersubjetividad, ya que su teoría se centra en la comunicación humana, elemento fundamental para el cuidado. (Cuadro No. 1)

\section{CUADRO No 1.}

\section{TEORÍAS SOCIOLÓGICAS BASADAS EN LA INTERSUBJETIVIDAD}

\begin{tabular}{|l|l|}
\hline \multicolumn{1}{|c|}{ Teoría } & \multicolumn{1}{|c|}{ Concepto de intersubjetividad } \\
\hline $\begin{array}{l}\text { Jürgen Hambermas } \\
\text { (1929) (Teoría de la acción Comunitaria) }\end{array}$ & $\begin{array}{l}\text { Un proceso de interacción, de un lado de la conciencia con el mundo y por el otro } \\
\text { de la conciencia con las otras conciencias. }{ }^{5}\end{array}$ \\
\hline $\begin{array}{l}\text { Alfred Schütz (1939) } \\
\text { (Teoría de la intersubjetividad) })\end{array}$ & $\begin{array}{l}\text { Se constituye entre y por los sujetos, se basa en la posibilidad no cuestionada } \\
\text { de ponerse en el lugar de otro, desde un aquí y un ahora determinados. }{ }^{6}\end{array}$ \\
\hline $\begin{array}{l}\text { Trevarthen } \\
\text { (1977) } \\
\text { (Teoría de la intersubjetividad) }\end{array}$ & $\begin{array}{l}\text { Mutuo reconocimiento del otro que emerge de manera natural a partir } \\
\text { de la experiencia de ser reconocido por el otro. }{ }^{7}\end{array}$ \\
\hline
\end{tabular}


Schütz toma como punto de partida para el análisis de su propuesta a Husserl y la metodología de Weber (sociología comprensiva) y analiza las relaciones intersubjetivas a partir de las redes de interacción social proponiendo una interrelación cara a cara con otro individuo dejando de ser uno y siendo uno con el otro. ${ }^{\text {h }}$ Schütz plantea un enfoque basado en la interacción, definida a partir de las relaciones intersubjetivas desde un aquí y un ahora determinado; en su propuesta, destaca el reconocimiento de las relaciones sociales donde se interactúa de forma intersubjetiva. Esta concepción la fundamenta como una interacción basada en tiempo y espacio como se observa en la siguiente figura.

En la figura $\mathrm{N}^{0} .1$ se expone de alguna forma como a toda

\section{Figura 1.}

Propuesta del sociólogo Schütz, para determinar la concepción intersubjetiva entre las personas.

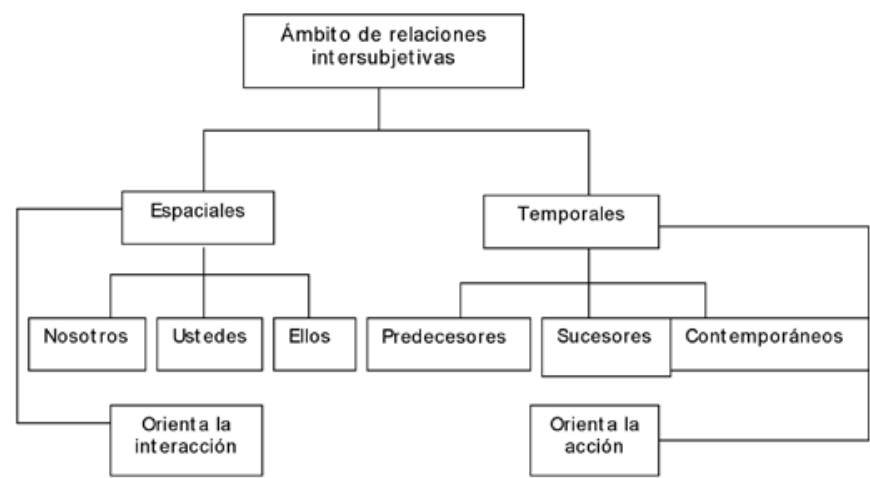

Fuente: Tomado de Guirola Lidia. Alfred Schütz y la pérdida de la inocencia en el análisis sociológico. Revista Casa del Tiempo. Difusión UAM. Junio 2000.

relación interpersonal le subyace una condición de espacio, que para el cuidado puede ser el hospital, la comunidad, su hogar, etc. y otra de temporalidad, es decir de tiempos para iniciar y concluir propósitos de interacción.

La intersubjetividad desde esta perspectiva se constituiría en entenderse mutuamente y compartir significados, aspecto que exige el cuidado humanizado, para entender comportamientos de las personas (movimientos, vocalizaciones, posturas, miradas, etc.) como signos de comunicación sobre su forma de entender la vida, la salud, la enfermedad y las formas de afrontamiento, entre otros aspectos.

La disciplina de Enfermería tiene una práctica social, misma que exige al profesional una interacción con seres humanos, donde tienen una subjetividad individual, un entorno específico, con conocimientos y expectativas diferentes de vida, que la misma Enfermera pone en juego al relacionarse o interactuar con la persona de cuidado.

\section{PERSPECTIVAS DE INTERSUBJETIVIDAD EN LA DISCIPLINA DE ENFERMERÍA}

El concepto interpersonal en el campo de la Enfermería sugiere reconocer un encuentro con el otro, tal cual lo sustentan las fuentes teóricas de H. Peplau, J. Paterson y L. Zderad, J. Travelbee, J. Watson y R. Parse.

Estas teóricas de Enfermería, se refieren al término intersubjetividad, cuando identifican la relación como proceso interpersonal de asistencia para entender la propia conducta y ayudar al otro, exaltando la empatía, la simpatía y la comprensión, como elementos importantes del fenómeno que se ponen en juego cuando de cuidado en Enfermería se habla.

Las teóricas, definen al cuidado desde su propia perspectiva basándose en una concepción humanística del mismo y se refieren a la intersubjetividad de manera implícita o explícita, a través de la relación Enfermera- persona, de tal forma que convergen en los siguientes aspectos: miran el fenómeno como proceso interpersonal, como medio para conocer la conducta del otro, en un proceso humano para comprender al otro y lograr un bienestar en él. (Cuadro No. 2)

\section{CONCLUSIONES}

Este trabajo permitió reflexionar en algunos elementos que están inmersos en el cuidado humanizado, lo que ofrece una herramienta para la construcción de la disciplina.

La relación Enfermera persona con enfoque humanista, no puede pasar por alto, el proceso intersubjetivo, ya sea visto como fenómeno, como elemento de comprensión ó como medio para brindar bienestar; cualesquiera que sea la denotación que se le dé a este proceso, permite ver a la persona de cuidado con una necesidad social, no solo con un problema de salud sino con una tendencia a un encuentro con alguien que escuche, espere, entienda, lo anime, intente conocer, y que a través de esto se mire en el otro, como ser susceptible de atención y cuidado.

No se puede desligar una buena práctica del cuidar de los valores humanistas, pues los cuidados Enfermeros tienen que ver con el ser humano, con la defensa de todas sus dimensiones, de toda su complejidad. 
Cuadro No. 2.

Teorías de Enfermería que abordan la relación Enfermera- paciente.

\begin{tabular}{|l|l|l|}
\hline \multicolumn{1}{|c|}{$\begin{array}{c}\text { Concepto Relación } \\
\text { Interpersonal }\end{array}$} & \multicolumn{1}{|c|}{ Punto Sustantivo } & \multicolumn{1}{c|}{ Características Comunes } \\
\hline $\begin{array}{l}\text { Hildergard Peplau } \\
\text { (1952) }\end{array}$ & $\begin{array}{l}\text { Consiste en ser capaz de entender la propia conducta } \\
\text { para ayudar a los demás a identificar sus problemas. }\end{array}$ & $\begin{array}{l}\text { Proceso interpersonal, que consta de } \\
\text { cuatro fases: orientación, identificación, } \\
\text { explotación, y resolución. }\end{array}$ \\
\hline $\begin{array}{l}\text { Josephine Paterson } \\
\text { y Loretta Zderad } \\
(1961)\end{array}$ & $\begin{array}{l}\text { Proceso de transacción intersubjetiva en el que las } \\
\text { necesidades relacionadas con la salud/enfermedad } \\
\text { son percibidas. }\end{array}$ & $\begin{array}{l}\text { Relación intersubjetiva, para lograr } \\
\text { bienestar y superación de la enfermedad.j }\end{array}$ \\
\hline $\begin{array}{l}\text { Joyce Travelbee } \\
(1964)\end{array}$ & $\begin{array}{l}\text { Proceso interpersonal de asistencia a la persona, } \\
\text { para prevenir enfermedad; el objetivo es encontrar } \\
\text { un significado a su experiencia y darle esperanza. }\end{array}$ & $\begin{array}{l}\text { Refuerza la empatía, la simpatía } \\
\text { y la comprensión, así como en los aspectos } \\
\text { emocionales de la Enfermería. }\end{array}$ \\
\hline $\begin{array}{l}\text { Jean Watson } \\
(1978)\end{array}$ & $\begin{array}{l}\text { Proceso intersubjetivo de humano a humano. } \\
\text { El objetivo es ayudar a la persona a conseguir el más } \\
\text { alto nivel de armonía entre su alma, cuerpo y espíritu. }\end{array}$ & $\begin{array}{l}\text { La Enfermera entra en el campo } \\
\text { fenoménico de otra persona para percibir } \\
\text { y sentir lo vivido por la otra persona.! }\end{array}$ \\
\hline $\begin{array}{l}\text { Rosemarie Rizzo Parse } \\
(1981)\end{array}$ & El cuidado está centrado en el ser humano. & $\begin{array}{l}\text { La Enfermera en relación intersubjetiva } \\
\text { con la persona o familia, permite describir } \\
\text { una situación vivida y comprender su } \\
\text { significado.m }\end{array}$ \\
\hline
\end{tabular}

\section{REFERENCIAS BIBLIOGRÁFICAS}

1 Domínguez - Alarcón; C. Para una sociología de la profesión de Enfermería en España. Revista Española de Investigaciones Sociológicas. No 8, 1979.

2 Watson; J. Adaptación y traducción: Beatriz Sánchez. Ciencia Humana y Cuidado Humano: Una teoría de Enfermería. National League for Nursing. 1988, pp. 31-36.

3 Rizo - García, M. Academia de Comunicación y Cultura, Universidad Autónoma de la Ciudad de México. Razón y palabra. Primera Revista Electrónica de Comunicación. No.24.

4 Morán - Aguilar; V., Mendoza - Robles; A. El intercambio de conocimiento sobre cuidado humano en Ibero América. Biblioteca Las casas, 2007; 3(1). Disponible en <http://www. index-f.com/lascasas/documentos/lc0199.php $>$ Consultado el 26-05-07.

5 Pontel; Horacio. Nietzsche en castellano, Heidegger en castellano. Revista Instantes y Azares. Disponible en: www.nietzscheana. com.ar/sobre_habermas.htm. Consultado el 15 Junio del 2007.

6 Guirola; Lidia. Laberinto. Alfred Shütz y la pérdida de la inocencia en el análisis sociológico. Revista Casa del Tiempo. Difusión UAM. Junio 2000. Disponible en: http://www.difusioncultural.uam.mx/revista/junio2000/girola.html Consultado por última vez 15 Junio 2007.
7 Trevarthen C. Descriptive analysis of Infant Comunicative en H.R. Schafter (ed). Studies in mother. Infant Interaccion. London. Academia. Press. 1977.

8 Pinto; N. Intersubjetividad, Comunicación y Cuidado. En: Dimensiones del Cuidado. Grupo de Cuidado. Facultad de Enfermería. Universidad Nacional de Colombia. 1998.

9 Marriner T. A. Modelos y teorías de Enfermería. $6^{a}$ ed. Ed. Elsevier, Mosby. España, 2007. p. 54.

10 Marriner T. A. Modelos y teorías de Enfermería. 4ª ed. Ed. Harcourt, España, 1998. p. 337.

11 Idem. p. 62.

12 Kérouac, S., Pepin, J., Ducharme, F. Duguette, A. Major, F. El pensamiento Enfermero. Masson. Madrid, 1996. Capítulo 2, p. 44.

13 Idem. p. 41.

\section{DIRECCIÓN PARA CORRESPONDENCIA}

Mtra. Rosa María Ostiguín M.: rosyostiguin@hotmail.com 